\title{
Static analysis of wind turbine blade using 1D FE model
}

\author{
${ }^{1 *}$ Tushar Sharma, ${ }^{2}$ V. Murari, ${ }^{3}$ K.K. Shukla \\ ${ }^{I^{*}}$ Application Engineer - FEA, ARK Infosolutions Pvt. Ltd., Noida, INDIA \\ ${ }^{2}$ Department of Applied Mechanics MNNIT Allahabad, Prayagraj, INDIA \\ ${ }^{3}$ Department of Civil Engineering, NIT Jamshedpur, Jharkhand, INDIA \\ "Corresponding Author: e-mail: Tushar.sharma@arkinfo.in, Tel +91-8860052577 \\ ORCID iDs: https://orcid.org/0000-0001-6249-9722 (Sharma), https://orcid.org/0000-0002-4697-5853 (Murari), https://orcid.org/0000-0002-7070-8712 (Shukla)
}

\begin{abstract}
The study presents a unique technique to determine the static response of wind turbine (WT) blade. A 1D Finite element (FE) beam model of WT blade is developed using thin-walled beam theory coupled with PreComp tool used to compute crosssectional stiffness with elastic coupling effects. A realistic $9.2 \mathrm{~m}$ long, WT blade is developed using different aerofoils with fourth order polynomial variation for twisting angle of blade span. Three different aerfoil sections NREL S818, NREL S825, and NACA 2412 are employed in the current study. For validation, the results of 1D model developed using MATLAB are compared with that of 3D WT blade model which is analyzed in ANSYS using NuMAD..
\end{abstract}

Keywords: Thin-walled beam; Wind turbine blade; PreComp; NuMAD; Finite element method.

DOI: http://dx.doi.org/10.4314/ijest.v13i1.19S

Cite this article as:

Sharma T., Murari V., Shukla K.K.. 2021. Static analysis of wind turbine blade using 1D FE model. International Journal of Engineering, Science and Technology, Vol. 13, No. 1, pp. 125-130. doi: 10.4314/ijest.v13i1.19S

Received: December 1, 2019; Accepted: February 5, 2021; Final acceptance in revised form: March 31, 2021

This paper was earlier presented at the International Conference on Energy, Environment \& Material Sciences (ICE2M), 1-3 December 2019 and substantially improved for this Special Issue. Guest Editor: Dr. Sri Niwas Singh, Professor (HAG), Department of Electrical Engineering, Indian Institute of Technology Kanpur, 208016 (U.P.) India, former Vice-Chancellor, Madan Mohan Malviya University of Technology Gorakhpur (April 2017 to July 2020).

\section{Introduction}

An in-depth understanding of the static responses of WT blades becomes necessary to design thinner and lighter WT blades effectively. Since blades are the primary components of the WT, the design of blades is a challenging task. El Chazly, 1993 proposed a complete 3D geometric and structural model of the blade using bending triangular plate finite elements for the determination of the maximum stresses and deflections that would be produced due to aerodynamic loading on the rotor blade. Locke and Valencia, 2003 developed a FE model to determine the static and buckling phenomena of the baseline and carbon hybrid design of blade. Vo and Lee, 2007 developed a model, applicable to thin-walled uniform box beam, subjected to a different boundary and loading conditions. Using 1D finite element, the effects of fiber angle, stacking sequence, and boundary conditions are studied. Liu and Ren, 2011 studied the vibration and stability of WT blades modelled as thin-walled closed beams, using extended Galerkein method based on variational asymptotic analysis. Bayoumy et al., 2013 formulated a continuum-based 3D FE model to investigate the transient response of the elastic WT blade having taper and twist into account. Fernandez et al., 2018 studied the distribution of local and global stress/strain of the WT blade under the action of aerodynamic loads. In the present work, the 1D FE thin-walled beam model for WT blade is modeled using. MATLAB and results are compared with the 3D model developed in ANSYS for the static response. 


\section{Mathematical Formulation}

To derive the 1D thin wall beam model following coordinate systems are used: (i) $\mathrm{x}, \mathrm{y}, \mathrm{z}$ global coordinate system, (ii) n, s, z local coordinate system. The above coordinate systems are related to the angle of rotation $\theta$ as shown in Figure 1.

The displacement field applied to the whole contour is expressed as (Vo and Lee, 2007):

$$
\begin{aligned}
& \bar{u}(s, z)=U(z) \sin \theta(s)-V(z) \cos \theta(s)-\Phi(z) q(s) \\
& \bar{v}(s, z)=U(z) \cos \theta(s)+V(z) \sin \theta(s)+\Phi(z) r(s) \\
& \bar{w}(s, z)=W(z)-U^{\prime}(z) x(s)-V^{\prime}(z) y(s)-\Phi(z) \omega(s)
\end{aligned}
$$

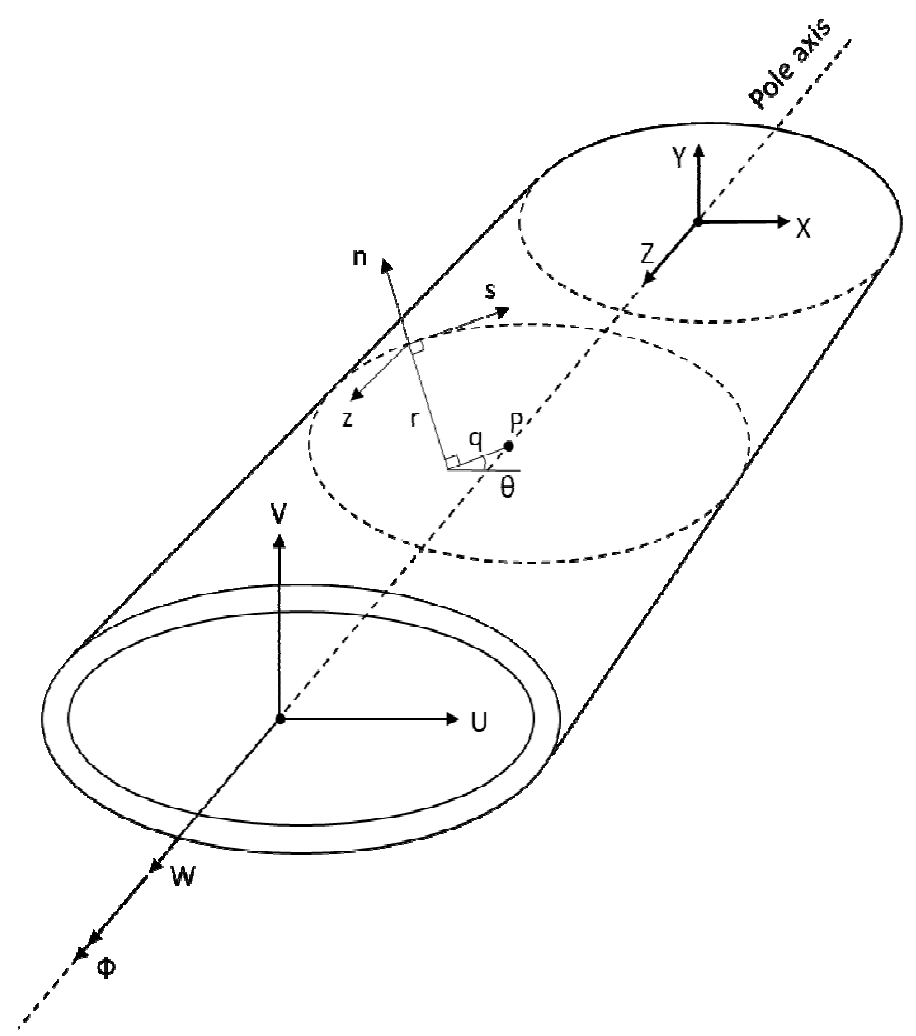

Figure 1. Coordinates system of thin walled beam

Where, $\mathrm{U}, \mathrm{V}, \mathrm{W}$ are the displacements of a point in $\mathrm{x}, \mathrm{y} \& \mathrm{z}$ directions, respectively. The warping function $(\omega)$ is expressed as:

$$
\begin{aligned}
& \omega(s)=\int_{s_{0}}^{s}\left[r(s)-\frac{F(s)}{\tau(s)}\right] d s \\
& \operatorname{as} \oint_{i} \frac{F(s)}{\tau(s)} d s_{i}=2 A_{i}
\end{aligned}
$$

Deformations at any point on profile are given in terms of mid-surface displacement by:

$$
\begin{aligned}
u(s, z, n) & =\bar{u}(s, z) \\
v(s, z, n) & =\bar{v}(s, z)-n \frac{\partial \bar{u}(s, z)}{\partial s} \\
w(s, z, n) & =\bar{w}(s, z)-n \frac{\partial \bar{u}(s, z)}{\partial z}
\end{aligned}
$$


The strain field based on small deformation theory is given by:

$$
\left.\begin{array}{l}
\varepsilon_{s}=\bar{\varepsilon}_{s}+n \bar{k}_{s} \\
\varepsilon_{z}=\bar{\varepsilon}_{z}+n \bar{k}_{z} \\
\gamma_{s z}=\bar{\gamma}_{s z}+n \bar{k}_{s z}
\end{array}\right\}
$$

where, $\bar{\varepsilon}_{s}, \bar{\varepsilon}_{z}, \bar{\gamma}_{s z}$ are mid-surface strains and $\bar{\kappa}_{s}, \bar{\kappa}_{z}, \bar{\kappa}_{s z}$ are mid-surface curvatures. Assuming that, the cross-section of thinwalled beam does not deform in its plane, i.e., $\bar{\varepsilon}_{s}$ and $\bar{\kappa}_{s}$ are neglected.

Thus, the resulting strains are;

$$
\begin{aligned}
& \varepsilon_{z}=\varepsilon_{z}^{o}+(x+n \sin \theta) k_{y}+(y-n \cos \theta) k_{x}+(\omega-n q) k_{\omega} \\
& \gamma_{s z}=\left(n+\frac{F}{2 \tau}\right) k_{s z}
\end{aligned}
$$

where $\varepsilon_{z}^{o}$ is axial strain, $k_{x}$ and $k_{y}$ are biaxial curvatures in $x \& y$ directions, respectively. $k_{\omega} \& k_{s z}$ are warping curvatures and twisting curvatures in beams and expressed as:

$$
\left.\begin{array}{l}
\varepsilon_{z}^{o}=W^{\prime} \\
k_{x}=-V^{\prime \prime} \\
k_{y}=-U^{\prime \prime} \\
k_{\omega}=-\phi^{\prime \prime} \\
k_{s z}=2 \phi^{\prime}
\end{array}\right\}
$$

By using the variational principle, the weak form is obtained as;

$$
0=\int_{0}^{l}\left(N_{z} \delta W^{\prime}-M_{y} \delta U^{\prime \prime}-M_{x} \delta V^{\prime \prime}-M_{\omega} \delta \phi^{\prime \prime}+2 M_{\tau} \delta \phi^{\prime}+q \delta V+t \delta U\right) d z
$$

where,

$$
\begin{aligned}
& N_{z}=\int_{A} \sigma_{z} d s d n \\
& M_{y}=\int_{A} \sigma_{z}(x+n \sin \theta) d s d n \\
& M_{x}=\int_{A} \sigma_{z}(y-n \cos \theta) d s d n \\
& M_{\omega}=\int_{A} \sigma_{z}(\omega-n q) d s d n \\
& M_{\tau}=\int_{A} \sigma_{s z}\left(n+\frac{F}{2 \tau}\right) d s d n
\end{aligned}
$$

Therefore, by using the constitutive equation for corresponding material and equation (8), the force-strain relations are obtained as:

$$
\left\{\begin{array}{l}
N_{z} \\
M_{y} \\
M_{x} \\
M_{\omega} \\
M_{\tau}
\end{array}\right\}=\left[\begin{array}{ccccc}
E_{11} & E_{12} & E_{13} & E_{14} & E_{15} \\
& E_{22} & E_{23} & E_{24} & E_{25} \\
& & E_{33} & E_{34} & E_{35} \\
& & & E_{44} & E_{45} \\
& & & & E_{55}
\end{array}\right]\left\{\begin{array}{l}
\varepsilon_{z}^{o} \\
k_{y} \\
k_{x} \\
k_{\omega} \\
k_{s z}
\end{array}\right\}
$$


where, $\left[\mathrm{E}_{\mathrm{ij}}\right]$ is the stiffness matrix depended on geometric and material properties.

In recent years, researchers have worked and developed many computation tools to determine the structural properties of WT blade. Among them, PreComp an open access code developed by NREL Laboratory is chosen for this present study. For the given geometry and material properties of the WT blade, PreComp evaluates the cross-sectional stiffness that is used as input to [ $\left.\mathrm{E}_{\mathrm{ij}}\right]$ in Eq.(9).

Utilizing the equation (5)-(9), the generalised form of equation of thin-walled composite box beam is obtained, and expressed as:

$$
\begin{aligned}
& E_{11} W^{\prime \prime}-E_{12} U^{\prime \prime \prime}-E_{13} V^{\prime \prime \prime}-E_{14} \phi^{\prime \prime \prime}+2 E_{15} \phi^{\prime \prime}=0 \\
& E_{12} W^{\prime \prime \prime}-E_{22} U^{i v}-E_{23} V^{i v}-E_{24} \phi^{i v}+2 E_{25} \phi^{\prime \prime \prime}+t=0 \\
& E_{13} W^{\prime \prime \prime}-E_{23} U^{i v}-E_{33} V^{i v}-E_{34} \phi^{i v}+2 E_{35} \phi^{\prime \prime \prime}+q=0 \\
& E_{14} W^{\prime \prime \prime}+2 E_{15} W^{\prime \prime}-E_{24} U^{i v}-2 E_{25} U^{\prime \prime \prime}-E_{34} V^{i v}-2 E_{35} V^{\prime \prime \prime}-E_{44} \phi^{i v}+4 E_{55} \phi^{\prime \prime}=0
\end{aligned}
$$

where, $\mathrm{q}$ and $\mathrm{t}$ are applied normal force, tangential force respectively. W, $\mathrm{U}, \mathrm{V}$ and $\Phi$ are not independent variables as they are fully coupled with each other.

\section{FE Formulation}

1D displacement based FE method is implemented for beam model, considering a combination of two interpolation function i.e. Lagrange interpolation function $\left(N_{j}^{L}\right)$ and hermite-cubic interpolation function $\left(N_{j}^{H}\right)$,

The generalized displacements over each element are expressed as:

$$
\begin{aligned}
W & =\sum_{i=1}^{n} w_{j} N_{j}^{L} \\
U & =\sum_{i=1}^{n} u_{j} N_{j}^{H} \\
V & =\sum_{i=1}^{n} v_{j} N_{j}^{H} \\
\phi & =\sum_{j=1}^{n} \phi_{j} N_{j}^{H}
\end{aligned}
$$

Substituting Eq. (11) into the governing differential equations (10), the FE model for a individual element can be written as;

$$
\left[K_{G}\right]\left\{\Delta_{e}\right\}=\left\{f_{e}\right\}
$$

Where $\left[\mathrm{K}_{\mathrm{e}}\right]=$ element stiffness matrix, $\left\{\Delta_{\mathrm{e}}\right\}=$ element displacement vector and $\left\{\mathrm{f}_{\mathrm{e}}\right\}=$ element force vector.

Then finally after assembly of these elements, we get global stiffness $\left[\mathrm{K}_{\mathrm{G}}\right]$ matrices and which can be written as:

$$
\left[K_{G}\right]\{\Delta\}=\{f\}
$$

Where, $\{\Delta\}=$ global displacement vector and $\{\mathrm{f}\}=$ global force vector.

\section{Results and Discussion}

To study the static response of 1D finite element model of WT blade a MATLAB code is prepared, 9.2m long WT blade is constructed with geometric and material properties. In the present study, the external and internal geometry, stacking sequence and material information of WTB (Locke and Valencia, 2003). Total span of the blade is separated into three areas, i.e. root, transition, and body. In the transition region, non-linear variation is considered where cross-sectional shape transforms from circular to aerofoil and in the body region, linear chord variation through the span is assumed with same aerofoil cross-section in the entire region (Figure 2). Twist distribution along the length of the blade taken in the present studies shown in Figure 3. At the tip of the 
blade, the angle of twist is negative, but as we move to the root, angle of twist increases with a different order of variation. The primary purpose of the shear web is to provide bending stiffness. Thus, the shear web is considered which is running from $1 \mathrm{~m}$ to $8.2 \mathrm{~m}$ of the blade length and is placed at $50 \%$ of the chord length at each station.

These geometric variations and material properties are provided as input to the PreComp which computes the station wise crosssectional stiffness for 1D beam element across the span of the blade. PreComp divides the blade in same numbers of stations in which $1 \mathrm{D}$ beam element is discretized while solving through FE method.

To have numerical validation for the present analytical solution and to assess the accuracy of the 1D WT blade miodel. The 3D model of WT blade is created in NuMAD with the same geometric and material properties as in 1D model. NuMAD creates WTB geometry which is imported to ANSYS where it is meshed using 3D shell element a shown in Figure 4.

For the analysis, the deformation of blades is computed considering different loading conditions: case 1: load act flapwise at the tip of the blade, Case 2: load act edgewise at the tip of the blade, Case 3: loads each of same magnitude applied flapwise at different location along the blade length for S818 aerofoil as shown in Table 1.

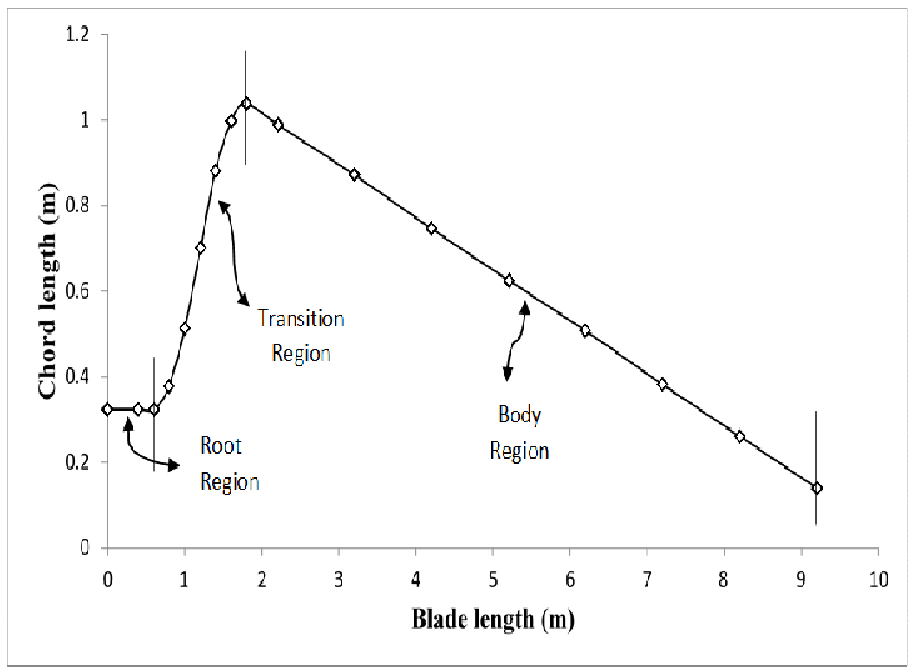

Figure 2. Chord variation along the span

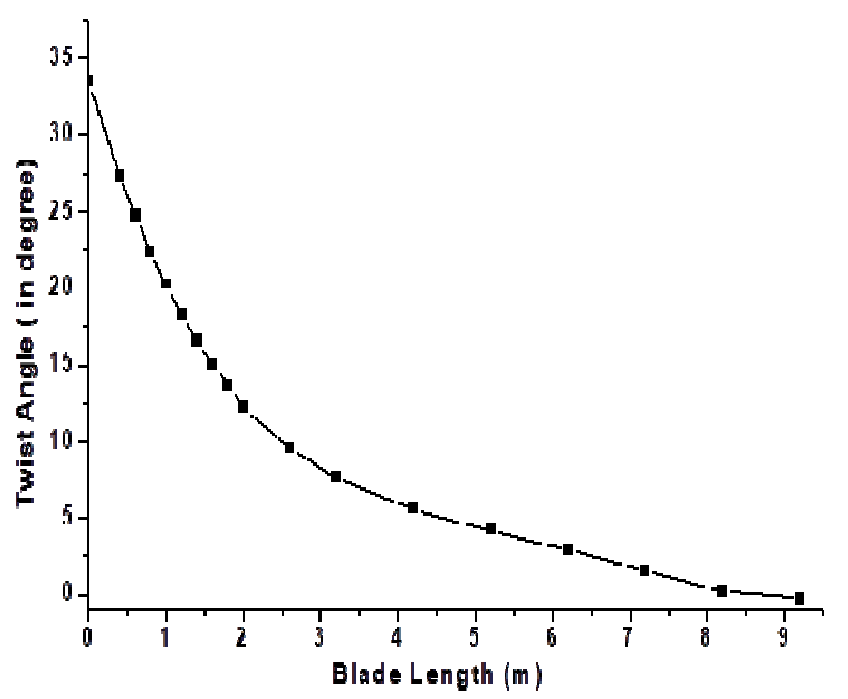

Figure 3. Twist variation along the span of WT blade

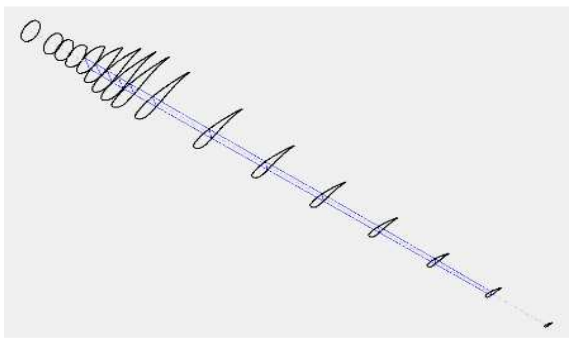

(a)

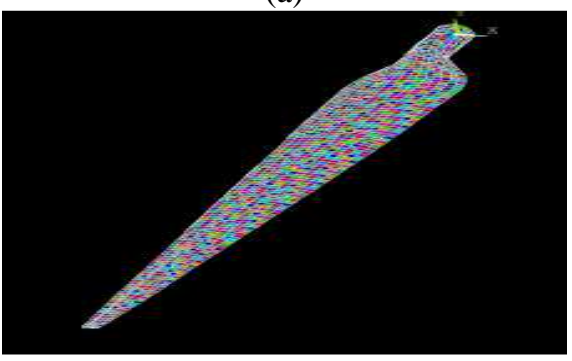

(d)

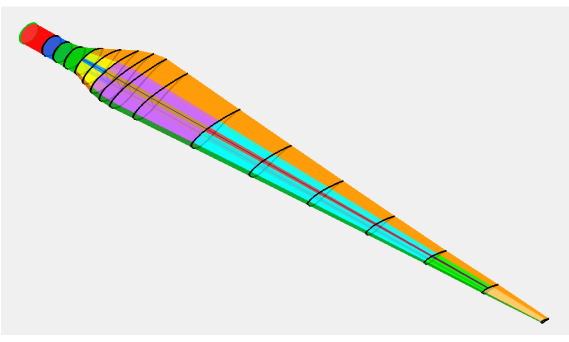

(b)

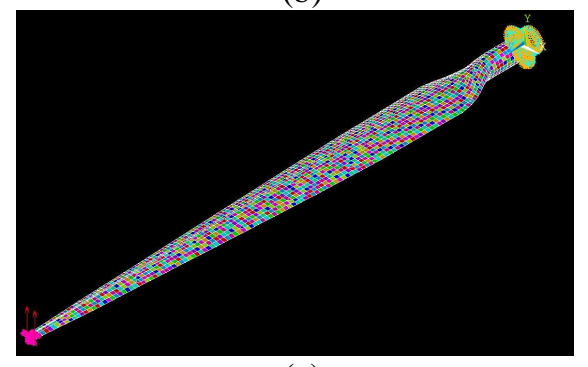

(e)

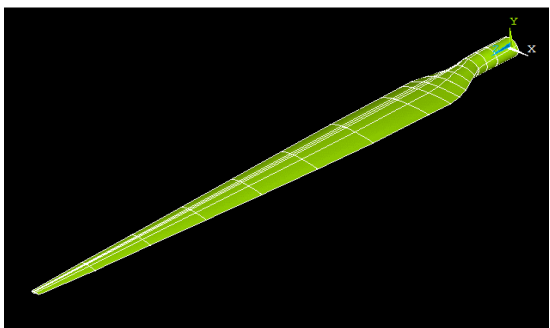

(c)

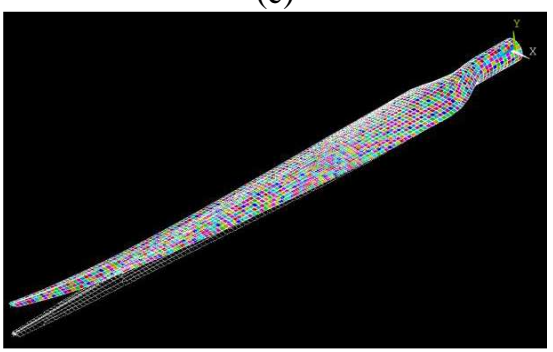

(f)

Figure 4. Complete analysis of 3D FE model of WTB using NuMAD and ANSYS 
Table 1. Comparison of the static response of a WT blade

\begin{tabular}{|c|c|c|c|c|c|c|c|}
\hline $\begin{array}{c}\text { Loading } \\
\text { Condition }\end{array}$ & \multicolumn{2}{|c|}{ 1D Beam Modal } & \multicolumn{2}{c|}{$\begin{array}{c}\text { 3D Modal } \\
\text { (ANSYS) }\end{array}$} & \multicolumn{2}{c|}{$\begin{array}{c}\text { Reference ( Locke and } \\
\text { Valencia, 2003) }\end{array}$} & $\begin{array}{c}\text { Difference } \\
(\%)\end{array}$ \\
\hline & $U_{x}(\mathrm{~mm})$ & $U_{y}(\mathrm{~mm})$ & $U_{x}(\mathrm{~mm})$ & $U_{y}(\mathrm{~mm})$ & $U_{x}(\mathrm{~mm})$ & $U_{y}(\mathrm{~mm})$ & \\
\hline Case 1 & 33.41 & 276.71 & 36.723 & 306.76 & 37.2 & 309 & $\approx 11$ \\
\hline Case 2 & 78.77 & 32.90 & 87.301 & 36.39 & 89.6 & 37.2 & $\approx 12$ \\
\hline Case 3 & 17.95 & 140.48 & 20.031 & 151.27 & - & 156 & $\approx 10$ \\
\hline
\end{tabular}

\section{Conclusions}

In this paper, a unique method to determine the static structural response of realistic WT rotor blade is presented. 1D FE thinwalled beam model of WT blade developed using thin-walled beam theory coupled with PreComp is studied. Effect of different aerofoil on the structural response of $9.2 \mathrm{~m}$ long realistic WT rotor blade is analyzed. For numerical validation, the results from the 1D model developed using MATLAB are compared with that of 3D model developed using NuMAD and analyzed in ANSYS. The results are found to be comparable and in good agreement with that of 3D model, which verifies that this unique methodology is suitable to determine the static response of WTB efficiently, fast, and accurately.

\section{Acknowledgement}

Would like to acknowledge the support from the Science and Engineering Research Board (SERB) for providing the fund (File no. MTR/2017/000586/MS) under MATRICS scheme.

\section{References}

Bayoumy A.H., Nada A.A., Megahed S.M. 2013. A continuum based three-dimensional modeling of wind turbine blades. Journal of Computational and Nonlinear Dynamics, 8(3). DOI: 10.1115/1.4007798

Bir G.S. 2006. User's guide to PreComp (pre-processor for computing composite blade properties) (No. NREL/TP-500-38929). National Renewable Energy Lab.(NREL), Golden, CO (United States).DOI: 10.2172/876556

El Chazly N.M., 1993. Static and dynamic analysis of wind turbine blades using the finite element method. Renewable energy, 3(6-7), pp.705-724.DOI: 10.1016/0960-1481(93)90078-U

Fernandez G., Usabiaga H., Vandepitte D. 2018. An efficient procedure for the calculation of the stress distribution in a wind turbine blade under aerodynamic loads. Journal of Wind Engineering and Industrial Aerodynamics, 172, pp.42-54.DOI: 10.1016/j.jweia.2017.11.003

Liu T., Ren Y. 2011. Vibration and flutter of wind turbine blade modeled as anisotropic thin-walled closed-section beam. Science China Technological Sciences, 54(3), pp.715-722. DOI: 10.1007/s11431-010-4230-y

Locke J., Valencia U., Ishikawa K., 2003. Design studies for twist-coupled wind turbine blades. In Wind Energy Symposium, Vol. 75944, pp. 324-331. DOI: 10.1115/WIND2003-1043.

Resor B.R. 2012. Numerical Manufacturing And Design Tool (NuMAD) (No. SAND2012-8679P). Sandia National Lab.(SNLNM), Albuquerque, NM (United States).

Vo T.P., Lee, J. 2007. Flexural-torsional behavior of thin-walled closed-section composite box beams. Engineering Structures, 29(8), pp.1774-1782.DOI: 10.1016/j.engstruct.2006.10.002

\section{Biographical notes}

Tushar Sharma received M. Tech. and Ph.D. from MNNIT Allahabad, India in 2013 and 2019, respectively. He is working as Application Engineer-FEA in Ansys division, ARK Infosolutions Pvt. Ltd. Noida, India. His research interests include composite material structures and application, computational mechanics, structural mechanics and Nano Mechanics problems. . He has published more than ten papers in referred international journals/conferences.

V. Murari received M.E. and Ph.D. from MIT, Anna University, India in 2004 and Indian Institute of Technology Kanpur, India in 2011, respectively. He is a Associate Professor in the Department of Applied Mechanic, MNNIT Allahabad, Prayagraj India. His research interests include Problems in Continuum Mechanics/Solid Mechanics, Continuum Damage Modelling/Damage Modelling of Composite Materials and Structures, Damage mechanics of repaired and recycled composites, Damage response of composite structures under dynamic loads, Correlation of energy absorption with damage propagation. He has published more than eighteen papers in referred international journals/conferences.

K.K. Shukla received M.E. and Ph.D. from MNNIT Allahabad, Prayagraj, India in 1988 and Indian Institute of Technology Delhi, India in 2001, respectively. He is a Professor in the Department of Applied Mechanic, MNNIT Allahabad, Prayagraj India. Presently he is on leave from MNNIT Allahabad and working Director, NIT Jamshedpur, India. His research interests include Composite Plates and Shells, Smart Structures, Retrofitting and Strengthening of RCC Structures, Computational Mechanics, Stability and Dynamics of structures and Multi-sclae Composites. He has published more than hundreds of papers in referred international journals/conferences. 